\title{
Introduction of fertilizers to the field surface
}

\author{
Sergey V. Belousov*, and Dmitry A. Filippov \\ Kuban State Agrarian University named after I.T.Trubilin, Krasnodar, Russian Federation
}

\begin{abstract}
The work is dedicated to the introduction of dry non-organic substances to the field surface by the new proposed distributor. The article has a research character, which is expressed in the analysis of methods and units of mechanization for applying mineral fertilizers, as well as excerpts from the works of leading scientists on the subject under study, the types of patents that ensure the operation of mineral fertilizer distributors, their shortcomings and ways to solve these problems. The main trend in the development of units for applying mineral fertilizers to improve the quality of their performance has been determined. The proposed concept of development of mechanization units for applying mineral fertilizers with a compact mechanized spreader is also justified. Its operation process is described. As a result of this work, the design of a compact mechanized distributor of mineral fertilizers is proposed and justified. The conclusions present the main results achieved so far.
\end{abstract}

\begin{abstract}
All over the world, agriculture is one of the main basic branches of the economy, which ensures the food security of the state. You can see the variety of agricultural crops cultivation in different countries of the world. Russian agriculture has been developing dynamically in the last 10-15 years. The volume of agricultural exports has been practically restored to the level of Soviet times. The domestic market is fully provided with its own grain, potatoes and nightshade vegetables, the issue of providing meat of cattle, pork and poultry should be resolved in the coming years [1].

The government of the Russian Federation is developing state programs aimed at solving existing problems in the course of modern trends in agricultural development and promising population growth. According to the Food and Agricultural Organization of the United Nations in recent years, more than one billion people suffer from hunger in the world and this figure is increasing year by year. According to the same organization by 2050, when the world population will be close to 10 billion people, there will be a reduction in fertile land and land suitable for agriculture [1,2]. Due to these forecasts, we can conclude that the demand for agricultural products such as cereals and vegetables will increase and the demand for animal forage will rise.

To increase the acreage, it will be necessary to master new methods of production: expansion of acreage in mountainous areas (for example, tiered galleries for rice and tea growing). The use of classical methods and units for mountainous area cultivation is not suitable. There is a need for more compact, mobile and productive specialized units of mechanization. Such equipments are power units with a capacity of up to $20 \mathrm{HP}$.
\end{abstract}

\footnotetext{
* Corresponding author: sergey belousov 87@mail.ru
} 
Leading scientists recommend an interdisciplinary approach to agricultural management that provides the rational use of natural resources and its adaptation to possible climate changes as a way to provide food in cities and suburbs. However, there is a problem of their aggregation with mounted units in their classical design [3].

Many designers improve productivity and machines by increasing power, speed, gripping width, weight reduction, combining technological operations and other modern units. But as it was said, this is not suitable for power units and the use of these units in conditions of limited land use and protective soil. It requires a radically new approach to the development of the area of crop cultivation.

One of the promising directions is the application of fertilizers to the soil surface. To implement this direction, it is necessary to study the researches of leading scientists in this direction, conduct patent searches for designs and identify the promising vector of the development in the approach to the implementation of this direction [4].

Thus, as a result of the patent analysis, a number of approaches were identified which were reflected in patents. Mineral fertilizer spreader, RU 2323563 C1 includes the body, running system, drive of working bodies, conveyor, dispersing units - fertilizer seeders, hydraulic system. Dispersing devices are made in the form of concentrically mounted two rotating disks with blades. The blades of the outer disk are equipped with extensions that repeat the shape of the blades and allow you to increase the length of the blade by $1 / 3$ of its length, depending on the selected width of spreading fertilizers. This technical solution allows you to ensure the uniformity of the fertilizer supply to the disks, preserve the fragmentation of fertilizers and increase the uniformity of distribution of fertilizers on the field surface [5,6].

Also, we have already known the work of the patent RU 2541393, according to which the mineral fertilizer seeder is designed for surface application of solid mineral fertilizers in a granular or crystalline form. The spreader (seeder) contains a hopper placed on the frame with a cover, safety grid and metering holes in the bottom, made with the possibility of adjusting the size by means of dampers, as well as a fertilizer spreader and a drive which works from the tractor's shaft capacity, and a mixing device, as well as additional working bodies that ensure the course of the process. A distinctive feature is that the fertilizer spreader is hung on the tractor's hitch and has a drive of the working bodies from the tractor's PTO [5,6].

Works under the patent RU 141387 U1, where the granulated mineral fertilizer spreader mounted on the vehicle frame which contains a conical hopper for introduction of mineral fertilizers, two dispersing disks with blades, a conveyor that feeds fertilizers from the hopper to the disks and gate valves installed in the rear part of the hopper and controlled by hydraulic cylinders or an electric actuator. The present spreader is made with steered front and rear wheels in which the torque transmission from the internal combustion engine to the wheels is effected through the transmission, the transfer case on the mid and rear hypoid gear, all wheels are made with double-wishbone suspension with air bag, four rear wheels contain individual planetary gearboxes, brakes of the disk type are installed on the four front wheels, conical hopper is closed by a removable cover, and also it includes a conveyor located below the hopper, two stainless steel dispersing disks with blades rotating in opposite directions, placed below the conveyor, and the drive of the rotation of the disks and the movement of the transporter is mechanical, consisting of gimbals and chain gears, or hydraulic, and the operator's cabin is equipped with a GPS navigator or GLONASS system, low-pressure tires are installed in all wheels. A distinctive feature is that the spreader is mounted on a self-propelled chassis [7]. 
These works and technologies have a number of disadvantages, design shortcomings, they cannot produce an accurate dosed uniform discharge of the material across the entire gripping width.

Works on patents have similar disadvantages: RU 144279 U1, RU 2343678 C2, RU 120840 U1, RU 2442308 C1.

Also, we have already known the manual fertilizer spreader, which contains a unit to move, a frame sustained by support wheels with unloading window, a hopper whose base is fixed to the frame, a centrifugal disk, a reducer whose output shaft is connected to the shaft of the centrifugal disk and an imbalance patent 143239 U1.

The disadvantages of this solution are the imperfection of the design, namely, the lack of opportunities to use it in conditions of limited land use, they cannot produce an accurate dosed and uniform spread of material across the entire gripping width [8].

We suggest using a small-sized spreader of dry non-organic mixtures in conditions of limited land use, which will significantly improve the quality of the resulting products and increase the productivity of this technological operation.

The technical result is achieved by the fact that in a mineral fertilizer spreader containing a unit for moving, a frame sustained by support wheels, a hopper with an unloading window, the base of which is fixed to the frame, a centrifugal disk, a reducer, whose output shaft is connected to the shaft of the centrifugal disk and an imbalance, an angular reducer is used according to the utility model, and as a unit for moving - tiller with front and rear portions while mounted on the rear hoist installed additional support wheel on which the imbalance is placed, protective screen is installed on the front of the tiller, and the horizontal shaft of the angular gear is connected with the shaft of the tiller, and the discharge window of the bunker is provided with a dispenser which is mounted with a cone-shaped fertilizer dispensers located on the centrifugal disk with radially mounted blades [9].

Mineral fertilizer spreader - figure 1 , it contains a unit for moving 1 , a frame 2 supported by support wheels 3 , a hopper 4 with an unloading window 5 , the base of which is fixed to the frame 2, a centrifugal disk 6 , an angular reducer 7 , the output shaft 8 which is connected to the shaft of the centrifugal disk 6 and an imbalance 9 on the rack 10. A motor cultivator (hereinafter referred to as a tiller 1) with front 11 and rear 12 attachments is installed in the spreader. On the rear hoist 11 an additional support wheel 13 is mounted on which the stand 10 with the imbalance 9 are placed on the front part of the tiller 1 , the protective screen 14 is installed and the horizontal shaft of the angular gear 15 is connected with the shaft of the power take-off (PTO) 16 of the tiller 1, with the discharge window of the bunker 4 which is provided with a spout 17 beneath which a cone-shaped fertilizer spreader 18 is mounted arranged on the centrifugal disk 6 , which has radially mounted blades 19.

The spreader works as follows: during the movement of the unit on the field, the fertilizer spreader mounted on the front hitch 11 of the motor cultivator 1 with a protective screen 14 is supported by the support wheels 3 , seed material naturally from the hopper 4 is poured through the spout 17 on the centrifugal disk 6, seed material through the fertilizer spreader 7 with a variable top angle is supplied to the blades 19 located on the centrifugal disk 6 and spread on the field surface. Centrifugal disk 6 is driven via the angular gear 7, by means of a chain or a belt drive, from support-drive wheels 3 or the PTO 16 of the tiller 1 . Imbalance 9 provides the ability to adjust continuously depending on the weight of seeds in the hopper 4 . To reduce the weight the metal hopper was made in a plastic frame with metal base. 


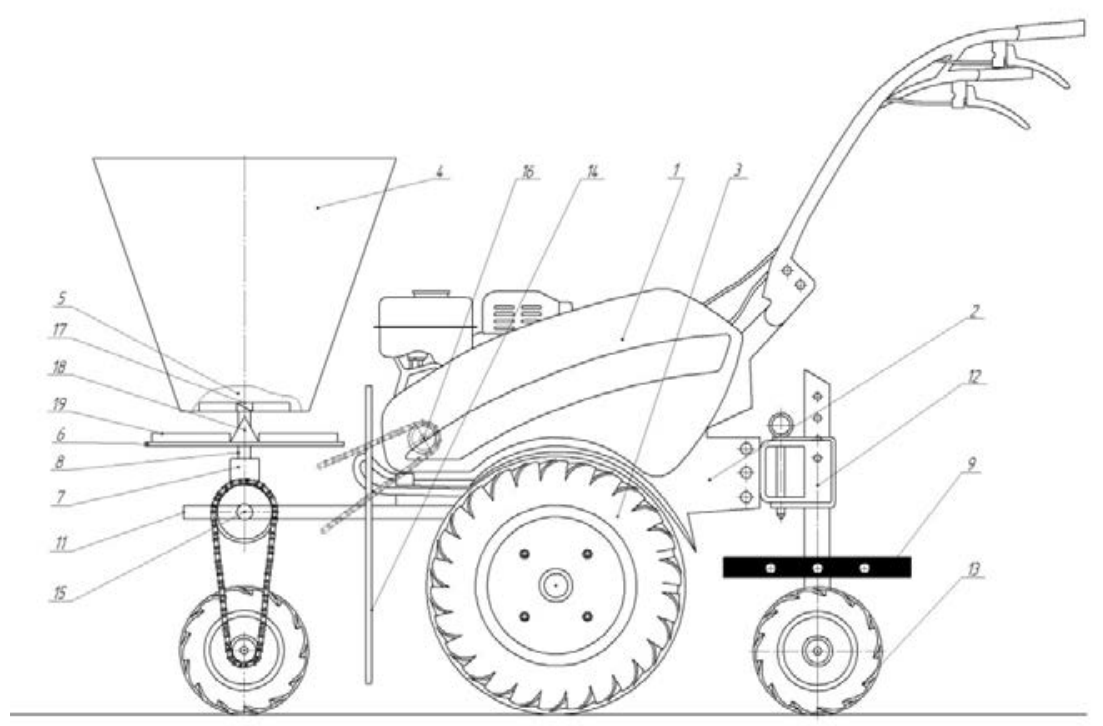

Fig. 1. Construction of the mineral fertilizer spreader.

A mineral fertilizer spreader containing a unit to move, a frame supported by support wheels, a hopper with an unloading window, the base of which is fixed to the frame, a centrifugal disk, a reducer whose output shaft is connected to the shaft of the centrifugal disk and an imbalance, an angular reducer, and as a unit to move - tiller with front and rear parts while the additional support wheel is mounted on the rear hoist where there is an imbalance, the protective screen is installed on the front of the tiller, and the horizontal shaft of the angular gear is connected with the shaft of the tiller, and the discharge window of the bunker is provided with a dispenser which is mounted in a cone-shaped fertilizer spreader placed on the centrifugal disk with radially mounted blades [10].

Technical solutions that are applied and implemented in this design due to the design features of the spreader, provide an increase in the productivity of applying dry inorganic mixtures in conditions of limited land use, which will significantly improve the quality of the resulting products and increase the productivity of this technological operation. The design is explained more detail in figure 1 , where the mineral fertilizer spreader is presented.

\section{References}

1. Belousov S.V., Belousova A.I., Experimental researches of plant protection means, MATEC Web of Conferences 2018. C. 05002.

2. Belousov S.V., Pomelyayko S.A., Novikov V.V., Design of the universal agricultural working body and study of its parameters, MATEC Web of Conferences 2018. C. 05006.

3. Belousov S.V., Gusak E.S., Theoretical justification of fruit separation process by a planetary fruit separator, E3S Web of Conferences 2019. C. 00056.

4. Bosoy E.S. Theory, construction and calculation of agricultural machines [Text] / E. S. Bosoy, I. I. Smirnov, O. V. Vernyaev, E. G. Sultan-Shah. - Moscow: "Engineering", 1978 - 568 p.

5. Belousova A.I., Moiseev A.V., Experimental researches of determination of degree of interaction of a working organ with the object of treatment in plant growing, MATEC Web of Conferences 2018. C. 05001. 
6. Goryachkin, V.P. Collection of ossions: in 3 t. / V.P. Goryachkin; by ed. action tsp VASHNIL prof. Dr. S.-H. Sciences ND Luchinsky. - 2nd ed. - M .: Kolos, 1968. - 455 p.

7. Belousova A.I., Moiseev A.V. Experimental researches of determination of degree of interaction of a working organ with the object of treatment in plant growing, MATEC Web of Conferences 2018. C. 05001.

8. Papusha S.K., Bogus A.E., Konovalov V.I., Interaction of rotary working body of roller type with the object of processing, MATEC Web of Conferences 2018. C. 05007.

9. Belousova A.I., Donchenko L.V., Automation of technological process of obtaining pectin, E3S Web of Conferences 2019. C. 00062.

10. Bogus A.E., Pusikova A.U., The thrashed mass kinematic parameters establishment in the roller thrashing unit clearance, E3S Web of Conferences 2019. C. 00036. 